\title{
Construction of quasipotentials for stochastic dynamical systems: An optimization approach
}

\author{
R. D. Brackston, ${ }^{1, *}$ A. Wynn, ${ }^{2}$ and M. P. H. Stumpf ${ }^{1,3}$ \\ ${ }^{1}$ Department of Life Sciences, Imperial College London, London SW7 2AZ, United Kingdom \\ ${ }^{2}$ Department of Aeronautics, Imperial College London, London SW7 2AZ, United Kingdom \\ ${ }^{3}$ School of BioScience and School of Mathematics and Statistics, University of Melbourne, Melbourne, Australia
}

(Received 21 May 2018; revised manuscript received 24 July 2018; published 29 August 2018)

\begin{abstract}
The construction of effective and informative landscapes for stochastic dynamical systems has proven a longstanding and complex problem. In many situations, the dynamics may be described by a Langevin equation while constructing a landscape comes down to obtaining the quasipotential, a scalar function that quantifies the likelihood of reaching each point in the state space. In this work we provide a novel method for constructing such landscapes by extending a tool from control theory: the sum-of-squares method for generating Lyapunov functions. Applicable to any system described by polynomials, this method provides an analytical polynomial expression for the potential landscape, in which the coefficients of the polynomial are obtained via a convex optimization problem. The resulting landscapes are based on a decomposition of the deterministic dynamics of the original system, formed in terms of the gradient of the potential and a remaining "curl" component. By satisfying the condition that the inner product of the gradient of the potential and the remaining dynamics is everywhere negative, our derived landscapes provide both upper and lower bounds on the true quasipotential; these bounds becoming tight if the decomposition is orthogonal. The method is demonstrated to correctly compute the quasipotential for high-dimensional linear systems and also for a number of nonlinear examples.
\end{abstract}

DOI: 10.1103/PhysRevE.98.022136

\section{INTRODUCTION}

Multidimensional nonlinear dynamical systems can exhibit complex behavior including limit cycles, strange attractors, and multiple fixed points. When also driven by stochastic perturbations, additional phenomena such as random switching between attractors and stochastic resonance may be observed [1]. The mathematics of such systems has its roots in the description of Brownian motion, which motivated the study of stochastic differential equations, generally written in the form

$$
d \boldsymbol{x}=f(\boldsymbol{x}) d t+g(\boldsymbol{x}) d \boldsymbol{W}_{t} .
$$

Here the deterministic component of the dynamics (the drift) is described by $f(\boldsymbol{x})$, while the stochastic component (diffusion) is given by $g(\boldsymbol{x}) d \boldsymbol{W}_{t}$, where the vector $d \boldsymbol{W}_{t}$ describes the increments of a Wiener process [2].

While models of Brownian motion describe the effect of exogenous disturbances on the motion of a particle [3], an important use of Stochastic Differential Equation (SDE) models is to capture the effect of endogenous disturbances or intrinsic noise. A key example of this is in chemical reaction systems [4,5], in which stochasticity arises due to the effect of a finite population of molecules. Similar finite-population-size effects can also arise in models of predator-prey dynamics [6] and neuronal population dynamics [7-9], to name just a few examples.

\footnotetext{
*r.brackston13@imperial.ac.uk
}

Published by the American Physical Society under the terms of the Creative Commons Attribution 4.0 International license. Further distribution of this work must maintain attribution to the author(s) and the published article's title, journal citation, and DOI.
In many applications, a popular description of SDE systems is in terms of an energy landscape, in which the dynamics are described as a ball moving in a potential basin. In some contexts, the landscape may simply give an intuitive description of the dynamics [10,11], while in others it may represent a true energy function [12]. In the context of developmental biology, Waddington's epigenetic landscapes provide a popular analogy for stem cell development $[13,14]$, even though it has proven impossible to define a true energy function for general developmental processes.

If correctly formed, landscapes may offer a quantitative analysis and interrogation tool beyond a merely phenomenological description, even in cases where the free energy of the system may not be defined. In particular, a so-called quasipotential landscape may be defined in terms of the FreidlinWentzell action functional [15]. This landscape provides quantitatively a measure of the relative stability of different states and the most probable paths between them. The concept of landscapes defined by the action has received particular recent attention [16], as has the development of methods to calculate the so-called minimum action path (MAP) [17-20]. Other recent methods have sought to evaluate quasipotential landscapes with a focus on the action functional. For linear systems an analytic expression for the quasipotential was obtained in Ref. [21], while numerical methods applicable to nonlinear systems have been given in Refs. [22-24]. While such methods may offer a numerical solution over a discretized space, in this work we present a method that generates an analytical solution and furthermore is applicable to both linear and nonlinear systems.

Given the motivation behind landscape descriptions, it is unsurprising that considerable effort has been applied to find other methods for landscape evaluation based either 
on experimental data or mathematical models $[25,26]$. In biological applications, the most popular and readily applied method is that based on the steady-state probability distribution [27]. This approach is based on the Fokker-Planck equation, which justifies computing a potential as $U(\boldsymbol{x}) \propto-\ln \left[P_{S}(\boldsymbol{x})\right]$. In practice the steady-state distribution is either obtained by solving the Fokker-Planck equation directly [28-30] or found from extensive simulations of the corresponding SDE [9,31-33]. While this method is easy to implement, it may be impractical for higher-dimensional systems and there is generally no guarantee that the derived landscape relates directly to the fixed points of the deterministic system or that a steady state distribution can even be obtained [34].

An alternative approach that solves some of these issues involves the "A-type" interpretation of the SDE $[35,36]$. In this approach a potential may be obtained by solving a set of PDEs, although this may often prove challenging [26].

Irrespective of how a landscape is obtained, its existence implies a decomposition of the deterministic vector field into two components, one of which is given by the gradient of the landscape while the other accounts for the remainder of the dynamics in $f(\boldsymbol{x})$ :

$$
f(\boldsymbol{x})=-\nabla U(\boldsymbol{x})+f_{U}(\boldsymbol{x}) .
$$

The construction of the landscape may therefore also be performed by considering the properties of this decomposition directly. As discussed in Ref. [15] and advocated in Ref. [37], one particularly interesting case is that in which the gradient and remainder are everywhere orthogonal, implying an independence between gradient-based and rotational dynamics. This is a property that may be satisfied in many cases, although exceptions do exist [38,39]. Motivated by this, we develop here a method to form landscapes satisfying the slightly more general suborthogonal condition that $\nabla U(\boldsymbol{x}) \cdot f_{U}(\boldsymbol{x}) \leqslant 0$, of which the orthogonal decomposition is a special case.

As we shall discuss below, the suborthogonal decomposition has the desirable property that under certain conditions it is directly related to the quasipotential. One of the other desirable properties of the landscape, and one that is satisfied by the suborthogonal decomposition, is that it should be a Lyapunov function [40] for the deterministic dynamics. In this context, Lyapunov functions are often used to prove the asymptotic stability of the fixed points $f(\boldsymbol{x})=0$. However, they also have the useful property that they provide the basins of attraction for the deterministic dynamics. There is therefore a strong equivalence between quasipotential landscapes and Lyapunov functions [41], suggesting that preexisting methods for generating such functions may also be used to obtain the quasipotential. This is the approach that we take here.

In this paper, we develop a method that performs a suborthogonal decomposition of the deterministic dynamics $f(\boldsymbol{x})$ by utilizing a popular method for the construction of Lyapunov functions: the sum-of-squares (SOS) method [42]. We will first provide some background on the properties of the suborthogonal decomposition in Sec. II before providing details of this computational method in Sec. III. We will then examine its application to a series of examples. The first of these examples will be linear systems in Sec. IV for which reference analytical solutions exist, followed by application to nonlinear systems

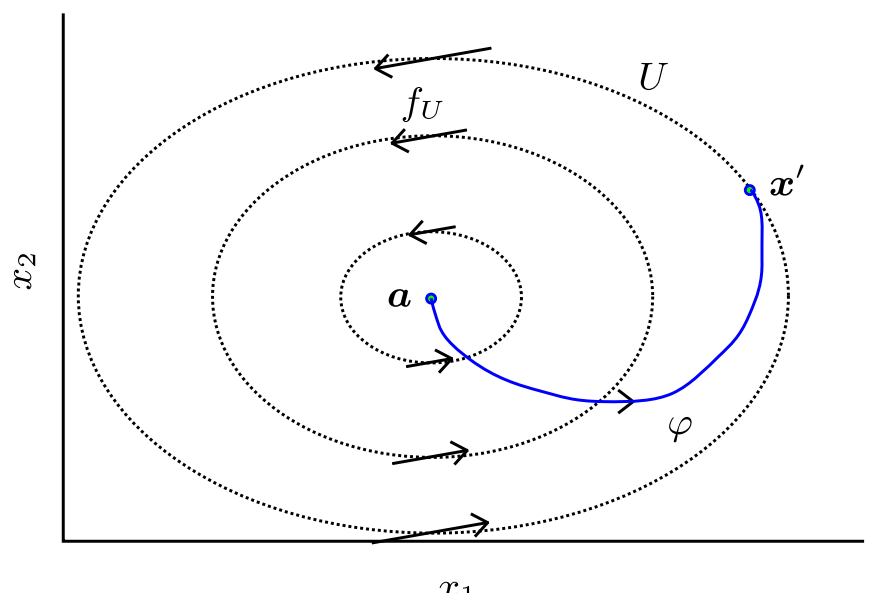

$x_{1}$

FIG. 1. A schematic of the suborthogonal decomposition and a minimum action path $\varphi$. The vector field $f_{U}$ always points either parallel to contours of $U$ or in a slightly "downhill" direction. The MAP is from a stable attractor at $\boldsymbol{a}$ to a point $\boldsymbol{x}^{\prime}$ within the basin of attraction.

with various properties in Sec. V. Conclusions will finally be given in Sec. VI.

\section{PROPERTIES OF THE SUBORTHOGONAL DECOMPOSITION}

In this work we consider the case in which the deterministic part of (1) is decomposed as in (2), and this decomposition has the property that

$$
\nabla U(\boldsymbol{x}) \cdot f_{U}(\boldsymbol{x}) \leqslant 0 .
$$

When this relation becomes an equality, the decomposition can be said to be orthogonal, while otherwise we will refer to the decomposition as suborthogonal. This property of the decomposition has a useful geometric interpretation: that the vector field $f_{U}$ is everywhere either parallel to the contours of $U$ or pointing in a slightly "downward" direction, as depicted in Fig. 1. As we shall now discuss, such a decomposition of the vector field both has a useful relation to the quasipotential and furthermore provides a Lyapunov function for the deterministic dynamics.

\section{A. The quasipotential}

Let us now restrict our analysis to systems in which the diffusion tensor $g(\boldsymbol{x})$ is a uniform diagonal matrix, describing purely additive noise,

$$
d \boldsymbol{x}=f(\boldsymbol{x}) d t+\sigma I_{n} d \boldsymbol{W}_{t} .
$$

Here $\sigma$ is a small constant and $I_{n}$ is the $n \times n$ identity matrix. Given such a system, the action associated with a path $\boldsymbol{x}(t)=\varphi$ is given by the following integral:

$$
S(\varphi)=\frac{1}{\sigma^{2}} \int_{0}^{T}\|\dot{\varphi}-f(\varphi)\|^{2} d t .
$$

Equation (5) is useful because in the limit, $\sigma \rightarrow 0$, the probability of the stochastic system following a certain path 
is directly related to the action of that path according to

$$
P(\varphi) \propto e^{-S(\varphi)} .
$$

Given the properties of the action, one can define a quasipotential $Q_{a}$ with respect to a fixed point $\boldsymbol{a}$ as

$$
Q_{a}\left(\boldsymbol{x}^{\prime}\right)=\inf _{\varphi, T}\left[S(\varphi) \mid \varphi(0)=\boldsymbol{a}, \varphi(T)=\boldsymbol{x}^{\prime}\right]
$$

This quasipotential therefore gives the action associated with the minimum action path to each point within the basin of attraction of the fixed point and in turn may be related to the probability distribution over the state space.

Given the suborthogonality condition of (3), the quasipotential may be evaluated as

$$
\begin{aligned}
Q_{\boldsymbol{a}}\left(\boldsymbol{x}^{\prime}\right)= & \frac{1}{\sigma^{2}} \inf _{\varphi, T}\left[\int_{0}^{T}\left\|\dot{\boldsymbol{x}}+\nabla U-f_{U}\right\|^{2} d t\right] \\
= & \frac{1}{\sigma^{2}} \inf _{\varphi, T}\left[\int_{0}^{T}\left\|\dot{\boldsymbol{x}}-\nabla U-f_{U}\right\|^{2}\right. \\
& \left.+4 \dot{\boldsymbol{x}} \cdot \nabla U-4 f_{U} \cdot \nabla U d t\right] \\
= & \frac{4}{\sigma^{2}}\left[U\left(\boldsymbol{x}^{\prime}\right)-U(\boldsymbol{a})\right] \\
& +\frac{1}{\sigma^{2}} \inf _{\varphi, T}[\int_{0}^{T} \underbrace{\left\|\dot{\boldsymbol{x}}-\nabla U-f_{U}\right\|^{2}}_{\text {(i) }}-\underbrace{4 f_{U} \cdot \nabla U}_{\text {(ii) }} d t] .
\end{aligned}
$$

In the orthogonal case, term (ii) is equal to zero, while the infimum of term (i) will approach zero for a path arbitrarily close to that satisfying the Ordinary Differential Equation (ODE) $\dot{\boldsymbol{x}}=\nabla U+f_{U} \cdot{ }^{1}$ Such a path may be proven to exist for the orthogonal decomposition, provided only that $\boldsymbol{x}^{\prime}$ is in the basin of attraction of $\boldsymbol{a}$ (see Appendix C). For the suborthogonal case the net contributions of both terms will be positive. The potential $U$ therefore provides a lower bound for the quasipotential according to

$$
Q_{a}\left(\boldsymbol{x}^{\prime}\right) \geqslant \frac{4}{\sigma^{2}}\left[U\left(\boldsymbol{x}^{\prime}\right)-U(\boldsymbol{a})\right] .
$$

In the case of the orthogonal decomposition this inequality becomes an equality.

\section{B. Lyapunov functions}

Lyapunov functions serve an important purpose in the field of nonlinear dynamical systems and feedback control, in proving asymptotic stability of a fixed point. For a system defined by a set of ODEs $\dot{\boldsymbol{x}}=f(\boldsymbol{x})$, we here define a Lyapunov function $U(\boldsymbol{x}): \mathbb{R}^{n} \rightarrow \mathbb{R}$ as one that satisfies the following constraints:

$$
\begin{aligned}
U(x) & \geqslant 0, \\
\nabla U(x) \cdot f(x) & \leqslant 0 .
\end{aligned}
$$

\footnotetext{
${ }^{1}$ Note that because $\boldsymbol{a}$ is a fixed point, such a path will take infinite time. However, given any small perturbation away from $\boldsymbol{a}$, the time will be finite.
}

These constraints impose the requirements that $U$ is everywhere positive and that $U$ decreases along trajectories of $f(\boldsymbol{x})$. The key implication of these constraints is that the Lyapunov function correctly captures the basins of attraction for all the stable fixed points: a region of the state space $\mathcal{A}$ around a fixed point $\boldsymbol{a}$ such that if $\boldsymbol{x}(0) \in \mathcal{A}, \boldsymbol{x}(t) \rightarrow \boldsymbol{a}$ as $t \rightarrow \infty$. Such a function therefore provides an accurate qualitative description of a (possibly multistable) landscape underlying the dynamics.

It is important to additionally note that conditions (9) are somewhat looser than in the typical definition for a Lyapunov function, in which additionally $U(0)=0$ and (9b) does not include equality. These more exacting requirement allow the function to be used to prove asymptotic stability, which our definition cannot. We choose our looser definition to allow the Lyapunov function to have wider interpretation as a multistable landscape that simply captures the basins of attraction.

Given the suborthogonal decomposition property of (3), we may rewrite constraint (9b) as

$$
\begin{aligned}
\nabla U \cdot f & =\nabla U \cdot\left(-\nabla U+f_{U}\right) \\
& =-\|\nabla U\|^{2}+\nabla U \cdot f_{U} \leqslant 0 .
\end{aligned}
$$

Provided that a sufficient constant offset is added to satisfy (9a), suborthogonality of the decomposition is therefore a sufficient condition for the potential $U$ to be a Lyapunov function for the deterministic dynamics described by $f$. We therefore use this condition in our optimization approach discussed below.

\section{THE OPTIMIZATION METHOD}

In this work we develop a method for landscape formation based on the sum-of-squares method for forming Lyapunov functions. We will therefore first give a brief overview of the standard algorithm through which SOS is used to find Lyapunov functions, before detailing the key changes required in our algorithm to achieve a suborthogonal decomposition. The algorithm itself has been implemented in the programming language Julia, built on the JuMP package for mathematical optimization $[43,44]$ and in MATLAB using the SOStools package [45].

\section{A. The standard SOS method}

Finding a Lyapunov function for nonlinear dynamical systems is generally a difficult problem [40,46] and is known to be NP-hard in the case where $U$ is a polynomial [47]: That is, given a possible function $U$, even checking that it satisfies the requirements may be very computationally expensive. The Lyapunov conditions of (9) are both conditions on the (semi-)positive definiteness of particular functions. The basis of the SOS method is that polynomial functions formed as the sum of the squares of lower-order polynomials are guaranteed to be positive definite and furthermore can be found via efficient optimization methods [42]. While the requirement that a function is SOS is stricter than that of positive definiteness, it makes the problem computationally tractable.

The standard use of SOS in forming a Lyapunov function involves expressing the Lyapunov conditions in terms of positive-definite polynomial constraints, which leads to 
solving the following:

$$
\begin{gathered}
\text { find a feasible }\left[c_{j}\right]_{j=0}^{m} \\
\qquad \begin{array}{c}
\text { subject to } \quad U=c_{0}+\sum_{j=1}^{m} c_{j} p_{j}, \\
U \geqslant \epsilon \sum_{i=1}^{n} x_{i}^{2}, \\
\nabla U \cdot f \leqslant 0 .
\end{array}
\end{gathered}
$$

Here the $p_{j}$ are a suitable set of $m$ monomial terms (e.g., $x_{1} x_{2}^{2}$ ) and the $c_{j}$ are real coefficients. Constraint (11a) therefore specifies that $U$ is a polynomial function. The parameter $\epsilon$ is a positive constant, typically of order one. Constraint (11b) therefore enforces (9a), ensuring that $U$ is positive. Finally, constraint (11c) enforces (9b) that $U$ should be a decreasing function along trajectories $f(\boldsymbol{x})$. What makes this a SOS problem is that the inequality constraints (11b) and (11c) both involve only polynomial functions. These constraints are therefore implemented as

$$
\begin{gathered}
U-\epsilon \sum_{i=1}^{n} x_{i}^{2} \in \Sigma, \\
-\nabla U(\boldsymbol{x}) \cdot f(\boldsymbol{x}) \in \Sigma,
\end{gathered}
$$

where $\Sigma$ denotes the set of polynomials that are sum-of-squares and therefore positive definite. In the following sections, similar constraints are also implemented in this way

The problem (11) is a pure feasibility problem and comes down to finding any $U$ composed of the monomials specified in (11a) and subject to the inequality constraints. There are therefore infinitely many possible solutions if $f(\boldsymbol{x})$ describes a stable system. As we will discuss below, we modify each of the steps of problem (11) to produce a convex optimization problem that yields a unique result.

\section{B. Towards orthogonality}

While the SOS method is able to generate Lyapunov functions for general $n$-dimensional dynamical systems, such functions are not unique and will generally not satisfy the suborthogonality requirement of (3). As noted by several previous authors [22,37,38], an orthogonality requirement results in a Hamilton-Jacobi equation $\nabla U \cdot f+\|\nabla U\|^{2}=0$ which is a nonlinear constraint on $U(\boldsymbol{x})$. Such constraints cannot be directly implemented into the SOS program. In order to implement such a constraint in a linear manner, consider the matrix

$$
M_{U}:=\left[\begin{array}{cc}
-\nabla U \cdot f & \nabla U^{\top} \\
\nabla U & I_{n}
\end{array}\right] \in \mathbb{R}^{(n+1) \times(n+1)} .
$$

A Schur complement argument [48] implies that $M_{U} \succeq 0$ is positive definite for any $\boldsymbol{x} \in \mathbb{R}^{n}$ if and only if

$$
-\nabla U \cdot f-\nabla U^{\top} I_{n} \nabla U \geqslant 0,
$$

which is equivalent to $\nabla U \cdot f_{U} \leqslant 0$, where $f_{U}$ is as defined in (2). This in turn implies that $\nabla U \cdot f \leqslant 0$, which satisfies the key requirement for a Lyapunov function and constraint (11c).
With the matrix inequality constraint in place, there will still be an infinite number of possible $U$, most of which will not come close to orthogonality. In order to find the unique $U$ that minimizes $\left\|\nabla U \cdot f_{U}\right\|$, we find it necessary to make $U$ as steep as possible, in this way maximizing the contribution of $-\nabla U$ to $f$. This is achieved by maximizing an appropriately chosen lower bound,

$$
B(\boldsymbol{x})=\sum_{i} \epsilon_{i} b_{i}(\boldsymbol{x}),
$$

where each $b_{i}$ is a monomial in $\boldsymbol{x}$ and the $\epsilon_{i}$ are real coefficients. The choice of these monomials is important and the method for choosing them is described below in Sec. IIIC. The complete optimization procedure is therefore as follows:

$$
\begin{aligned}
\underset{\left[c_{j}\right],\left[\epsilon_{i}\right]}{\operatorname{maximize}} & \sum_{i} \epsilon_{i} \\
\text { subject to } U & =c_{0}+\sum_{j=1}^{m} c_{j} p_{j}, \\
U & \geqslant \sum_{i} \epsilon_{i} b_{i}, \\
M_{U} & \succeq 0 .
\end{aligned}
$$

Compared with (11), one can see that the function $U$ is constructed in the same way as the sum of a set of monomial terms. The lower bound of (11b) has now been replaced with that of (16c), while (11c) has been replaced with the matrix constraint of (16d).

For the case that $f(\boldsymbol{x})$ is linear, this method can be proven to yield the correct result, as detailed in Appendix A.

\section{Choosing the monomial basis and lower bound}

The first key choice that must be made is that of the monomial basis from which $U$ is constructed, i.e., the $p_{j}$ in (16b). In the typical application of SOS to form Lyapunov functions, a candidate function is formed from a full collection of the monomials in $\boldsymbol{x}$ up to a given (even) degree $d$ [49]. For a given $n$-dimensional system, this results in $m=\left(\frac{n+d}{d}\right)$ monomials forming the polynomial function $U$ [50]. For example, for a fourth-order polynomial and four-dimensional state space this results in 70 monomials. Since the computational cost of the optimization increases with $m$, it is advantageous to reduce the basis if possible by exploiting particular properties of the function we are trying to obtain.

\section{A minimal basis}

We can initially provide an upper bound on the degree $d$ using the suborthogonality condition (3), which may be rewritten as

$$
\nabla U \cdot f+\|\nabla U\|^{2} \leqslant 0 .
$$

Since $\|\nabla U\|^{2} \geqslant 0$, the above inequality can only hold if the degree of $\nabla U \cdot f$ is at least as big as the degree of $\|\nabla U\|^{2}$. If $d$ is the degree of $U$ and $e$ the degree of $f$, then $d+e-1 \geqslant$ $2 d-2$, meaning that

$$
d \leqslant e+1 .
$$


While (18) provides an upper bound on the degree of $U$, we may further refine the basis (16b), motivated by the properties in the pure gradient case, in which $f_{i}=-\partial U / \partial x_{i}$. The minimal basis for such a $U$ is therefore provided by

$$
\left[p_{j}\right]_{j=1}^{m}=\mathrm{M}\left[\sum_{i} f_{i} x_{i}\right],
$$

where the operator $\mathrm{M}$ extracts a vector of monomials from a polynomial.

As an example, consider the vector field defined by

$$
f(\boldsymbol{x})=\left[\begin{array}{c}
x_{1}-x_{1}^{3} \\
-x_{2}^{3} \\
-x_{3}^{3}
\end{array}\right] .
$$

Here the highest order in $f$ is three so (18) implies that we will likely require a $d=4$ order polynomial. Given that the system has $n=3$ dimensions, the standard method leads to 35 monomials. By contrast, a minimal basis would only include five terms, $\left[x_{1}^{4}, x_{2}^{4}, x_{3}^{4}, x_{1}^{2}, 1\right]$.

\section{The lower bound}

Given a monomial basis for $U$, it is next necessary to choose the monomials $b_{i}$ in the lower bound (15). This choice is best illustrated via the following simple system described by

$$
\begin{aligned}
f(x) & =x-x^{3}+\beta \\
& =-\frac{d}{d x}\left[\frac{1}{4} x^{4}-\frac{1}{2} x^{2}-\beta x+C\right] .
\end{aligned}
$$

Given that (21) is one dimensional and therefore may be written as a pure gradient system, the minimal basis argument of (19) correctly ascertains that $U(x)$ will be composed only of monomials $\left[x^{4}, x^{2}, x, 1\right]$. A sensible choice for the lower bound will be $b(x)=x^{q}$, where $q$ is an even integer. The inequality constraint (16c) therefore becomes

$$
c_{3} x^{4}+c_{2} x^{2}+c_{1} x+c_{0} \geqslant \epsilon x^{q},
$$

where the choice of $q$ is of critical importance. In particular it must satisfy two particular properties.

a. Sufficient pressure. Suppose that we take $q=2$, now for any $c_{3}, c_{0}>0$ the constraint may be satisfied. Yet $c_{3}$ may remain arbitrarily small and the problem will not have converged. The lower bound is therefore unable to exert sufficient "upward pressure" on $U$.

b. Feasibility. Alternatively, consider $q=6$. In this case it is impossible to find coefficients such that $U>\epsilon x^{6}$, since as $x \rightarrow \infty, c_{3} x^{4}<\epsilon x^{6}$ for any $c_{3}, \epsilon>0$. The program will therefore be infeasible.

The correct choice for $q$ in this case is $q=4$, i.e., the highest degree in the basis for $U$. An example of this lower bound in practice is displayed in Fig. 2. The same principles of sufficient upward pressure and feasibility also apply to higherdimensional systems, such that the lower bound is chosen to consist of the highest degree single and mixed monomials in the basis for $U$.

\section{Extending the basis}

A correctly chosen "minimal" basis will naturally work for the case that $f(\boldsymbol{x})$ is a pure gradient system and may sometimes
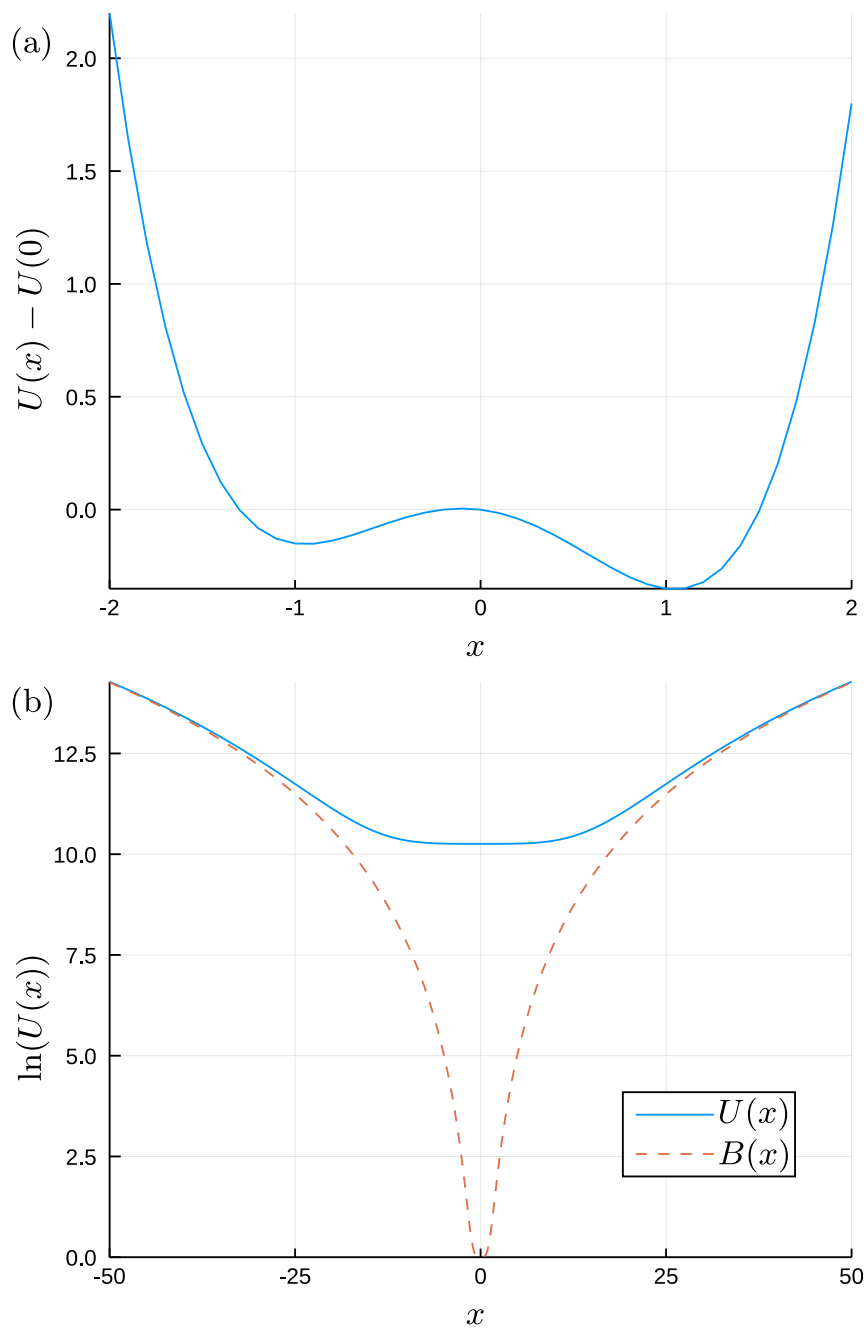

FIG. 2. A schematic of the lower bounding method. (a) The potential function in the region of the fixed points and (b) a comparison between the potential and lower bound.

work in other cases. ${ }^{2}$ However, more generally, our constraints may require that $\nabla U$ contains monomials beyond (19), as will be illustrated in later examples. Nonetheless, in addition to considerations of computational cost, the sensitivity of the method to the choice of lower bound means that we cannot simply choose all monomials up to degree $d$, as is done in the standard SOS method.

Consider again the example given in (21). Based on the above discussion it is clear that we could not have included an additional higher-order monomial in the basis for $U$. For example, had we included a $x^{6}$ term, we would logically have also chosen $q=6$ for the lower bound. Since the actual potential need not include a $x^{6}$ term, this would make the program infeasible. The principles of sufficient pressure and feasibility in the choice of the lower bound, therefore guide which monomial terms are permitted in the basis for $U$. The procedure for determining the basis is therefore as follows:

\footnotetext{
${ }^{2}$ For a linear system in which the matrix $A$ is normal, a minimal basis will also be sufficient since sparsity of the matrix is maintained through (28).
} 
(1) The highest-order monomial in any individual $x_{i}$ should be that obtained from the minimal basis.

(2) Other mixed monomials are required, provided that

(a) the total order is not more than the highest total order in the minimal basis,

(b) the individual order in each $x_{i}$ is not more than the highest individual order monomial in the minimal basis.

\section{Iterative improvement}

The process outlined above performs well for many systems but may require further improvement to push the obtained landscape closer to orthogonality. Given an initial Lyapunov function $U_{1}$ such as that found by implementing (16), one can attempt to iteratively improve the solution, generating at each step a second Lyapunov function $U_{2}$ composed of the same monomial basis. Each iteration involves solving the following optimization problem:

$$
\begin{aligned}
\underset{\left[c_{j}\right], \alpha, \epsilon}{\operatorname{minimize}} & \alpha \\
\text { subject to } U_{2} & =c_{0}+\sum_{j=1}^{m} c_{j} p_{j}, \\
U_{2} & \geqslant \epsilon \sum_{i} x_{i}^{2}, \\
M_{U_{2}} & \geq 0, \\
\nabla U_{2} \cdot\left(f+2 \nabla U_{1}\right) & \geqslant \alpha\left(f \cdot \nabla U_{1}\right)+(1+\alpha)\left\|\nabla U_{1}\right\|^{2}, \\
\alpha, \epsilon & >0 .
\end{aligned}
$$

The key to this optimization is that by using the initial guess, $U_{1}$, the optimization is constructed to be linear in the new improved Lyapunov function, $U_{2}$. Constraints (23c) and (23d) simply mirror those in optimization (16), namely positive definiteness of the Lyapunov function and the suborthogonality condition. Constraint (23e) is chosen such that for $\alpha=1$ equality is guaranteed if $U_{2}=U_{1}$, while for $\alpha<1$, $\nabla U_{2} \cdot f_{U_{2}} \leqslant \nabla U_{1} \cdot f_{U_{1}}$. This result is proven in Appendix B; however, the key point is that the optimization has a guaranteed feasible solution with $\alpha=1$ and $U_{2}=U_{1}$, while if a smaller $\alpha$ is obtained, then the new Lyapunov function is closer to orthogonality.

The complete method for obtaining the potential landscape therefore consists of first applying procedure (16), followed by a number of iterations of procedure (23). We generally find that only one or two iterations of this method are required for satisfactory convergence. The number of iterations may therefore either be prespecified or determined based on a convergence criterion.

\section{THE LINEAR CASE}

A special case of the orthogonal decomposition may be considered when the deterministic system is linear [22]. While this may seem a very limiting test case, the linear situation can exemplify some of the key scenarios and limitations that also apply in the nonlinear case, while remaining tractable to analysis.

\section{A. Properties of linear systems}

Linear dynamics may be written in terms of a matrix multiplication as

$$
\dot{\boldsymbol{x}}=f(\boldsymbol{x})=A \boldsymbol{x},
$$

where the matrix $A \in \mathbb{R}^{n \times n}$. The construction of the quasipotential then comes down to decomposing the matrix $A$ into two parts as

$$
A=A_{g}+A_{c},
$$

where the gradient matrix $A_{g}$ is a symmetric matrix that gives the potential according to

$$
U(\boldsymbol{x})=-\frac{1}{2} \boldsymbol{x}^{\top} A_{g} \boldsymbol{x} .
$$

Because $A_{g}$ is symmetric, it has purely real eigenvalues, while if the decomposition is orthogonal, the remainder matrix $A_{c}$ has purely imaginary eigenvalues and is such that the product $A_{g} A_{c}$ is an antisymmetric matrix. The potential matrix therefore describes the stability properties of the underlying system, while the curl component provided by $A_{c}$ describes purely oscillatory dynamics.

In linear cases it has been demonstrated that an orthogonal decomposition does always exist [51] and furthermore may be provided by an analytical expression [21] as

$$
A_{g}=\frac{1}{2}\left(\int_{0}^{\infty} e^{A t} e^{A^{*} t} d t\right)^{-1} .
$$

If the matrix $A$ is normal $\left(A A^{*}=A^{*} A\right)$, then this expression simplifies to

$$
A_{g}=\frac{1}{2}\left(A+A^{*}\right) .
$$

Since these expressions provide solutions against which our method can be tested, linear systems give a perfect test case for our optimization-based approach which does not rely on any prior knowledge of the properties of the system.

It is worth also noting here that normality of the matrix most typically arises when $A$ is a symmetric matrix, in which case the system has only a gradient component and $A_{c}=0$. For many other systems the matrix will be non-normal. ${ }^{3}$ In such cases it is generally understood that even when the eigenvalues describe a stable system, the non-normality, quantified by metrics such as the reactivity [52-54], leads to transient growth under the influence of perturbations. As has been noted before [9], expressions (27) and (25) imply that non-normal systems necessarily contain a curl component in order to fully describe the dynamics.

\section{B. A three-dimensional example}

We consider now the test-case of a three-dimensional linear system defined by

$$
\begin{aligned}
f(\boldsymbol{x}) & =A \boldsymbol{x} \\
& =\left[\begin{array}{ccc}
-5.0 & 0.0 & 0.2 \\
0.0 & -1.5 & 3.0 \\
0.5 & -5.0 & -1.0
\end{array}\right] \boldsymbol{x} .
\end{aligned}
$$

\footnotetext{
${ }^{3}$ It is important to note that while symmetry implies normality of the matrix, this is not always true the other way around.
} 
Implementation of methods (16) and (23) provides the following decomposition for $A$ :

$$
\begin{aligned}
A= & \underbrace{\left[\begin{array}{ccc}
-5.01 & 0.14 & 0.18 \\
0.14 & -1.55 & -0.02 \\
0.18 & -0.02 & -0.94
\end{array}\right]}_{A_{g}}, \\
& +\underbrace{\left[\begin{array}{ccc}
0.01 & -0.14 & 0.02 \\
-0.14 & 0.05 & 3.02 \\
0.32 & -4.98 & -0.06
\end{array}\right]}_{A_{c}},
\end{aligned}
$$

giving the quasipotential as

$$
\begin{aligned}
U(\boldsymbol{x})= & 2.50 x_{1}^{2}+0.78 x_{2}^{2}+0.47 x_{3}^{2} \\
& -0.14 x_{1} x_{2}-0.18 x_{1} x_{3}+0.02 x_{2} x_{3} .
\end{aligned}
$$

As can readily be observed, the matrix $A_{g}$ is symmetric and can easily be verified to have purely real (negative) eigenvalues. The matrix $A_{c}$ is not itself either symmetric or antisymmetric but can be verified to have purely imaginary eigenvalues. The dynamics associated with $A_{c}$ are therefore those of pure oscillation and evolve on level sets of the potential $U$.

It is noteworthy that for the system defined by $A$, there is no direct connection between $x_{1}$ and $x_{2}$. One may therefore naively expect such a direct connection to also be absent in the potential. Nevertheless, in order to provide an orthogonal decomposition, the potential matrix $A_{g}$ does include such terms that are then negated in $A_{c}$. This confirms that a truly minimal basis is indeed insufficient for forming the quasipotential, as discussed in Sec. IIIC3.

\section{Scaling with system size}

While the method may be verified to work for this threedimensional example, it is also useful to assess the accuracy and efficiency of the algorithm for higher-dimensional systems. We do this by randomly generating real, negative-definite matrices of varying size $n=2: 10$. In all such cases the method is able to perform a decomposition that satisfies the orthogonality constraint. Results for the computational cost are shown in Fig. 3, displayed on a logarithmic scale. It is evident that the cost increases considerably with system size, most likely in a factorial fashion. For small systems the algorithm converges in less than a second, while for larger systems it may take up to an hour.

It is worth noting here that some of the additional computational cost associated with larger systems arises from a greater number of times that the iteration procedure (23) must be applied, given a desired level of orthogonality. For lowdimensional problems we find that sometimes no iterations are required, while for systems of dimension $n=10$, up to five iterations may be necessary to achieve the same quality of result.

\section{THE NONLINEAR CASE}

Having studied the ability of our method to obtain the decomposition in linear cases, we now move on to fully

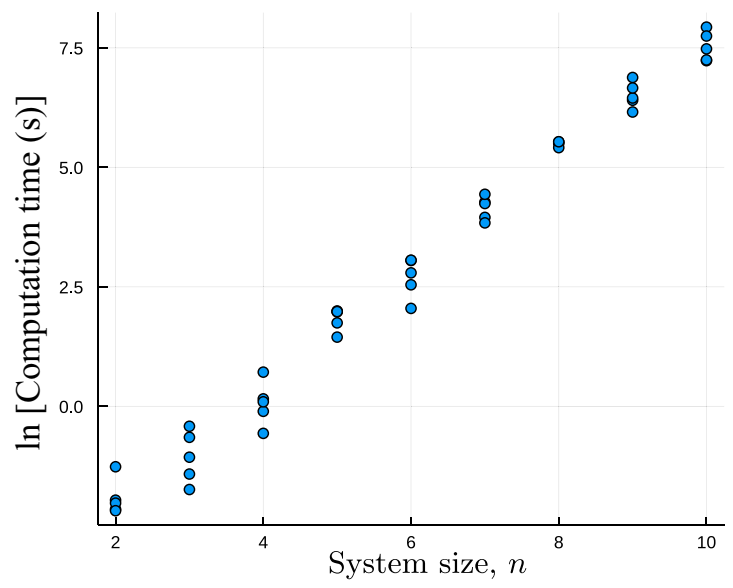

FIG. 3. Scaling of the computational cost with system size. Tests were performed on a basic desktop PC with 16 GB RAM and an Intel Core i3 processor.

nonlinear examples for which analytical solutions do not always exist.

\section{A. A nonlinear multistable system}

Our first example is the quartic system from Ref. [37] with four attractors and a known orthogonal decomposition. The dynamics are defined by

$$
f(\boldsymbol{x})=\left[\begin{array}{l}
-1+9 x_{1}-2 x_{1}^{3}+9 x_{2}-2 x_{2}^{3} \\
1-11 x_{1}+2 x_{1}^{3}+11 x_{2}-2 x_{2}^{3}
\end{array}\right],
$$

for which the true potential is given by

$$
U(\boldsymbol{x})=0.5\left(x_{1}^{4}+x_{2}^{4}\right)-5\left(x_{1}^{2}+x_{2}^{2}\right)+x_{1} x_{2}+x_{1} .
$$

For such a problem our method finds the potential to within five significant figures within a few seconds. Because the output of the algorithm is a symbolic expression for the potential, the actual value of $U$ may then be evaluated at any required points in the space with minimal further effort. This is in contrast of course, to methods that solve the associated PDE over a discretized grid, since evaluation outside of the solved area requires significant further computation.

\section{B. The Maier-Stein model}

We next apply the method to the widely studied Maier-Stein model in Ref. [55], defined by

$$
f(\boldsymbol{x})=\left[\begin{array}{c}
x_{1}-x_{1}^{3}-\gamma x_{1} x_{2}^{2} \\
-\mu\left(1+x_{1}^{2}\right) x_{2}
\end{array}\right] .
$$

This model has the property that when $\gamma=\mu$, the dynamics can be expressed in terms of a pure potential ${ }^{4} f(x)=-\nabla U$, where

$$
U(\boldsymbol{x})=\underbrace{+0.25}_{c_{1}} x_{1}^{4} \underbrace{+0.5 \mu}_{c_{2}} x_{1}^{2} x_{2}^{2} \underbrace{-0.5}_{c_{3}} x_{1}^{2} \underbrace{+0.5 \mu}_{c_{4}} x_{2}^{2} .
$$

\footnotetext{
${ }^{4}$ This may be verified by checking that $\frac{\partial f_{1}}{\partial x_{2}}=\frac{\partial f_{2}}{\partial x_{1}}$.
} 
TABLE I. Coefficients obtained for the Maier-Stein model for three different parameter combinations.

\begin{tabular}{rrrccc}
\hline \hline$\mu$ & \multicolumn{1}{c}{$\gamma$} & $c_{1}$ & $c_{2}$ & $c_{3}$ & $c_{4}$ \\
\hline 1.0 & 1.0 & 0.2499999 & 0.4999999 & -0.5000001 & 0.4999998 \\
1.0 & 10.0 & 0.1600785 & 1.0005570 & -0.3199390 & 0.0003017 \\
2.5 & 1.0 & 0.2495550 & 0.5109047 & -0.4991115 & 1.2496490 \\
\hline \hline
\end{tabular}

For cases in which $\gamma \neq \mu$, the system also has a nongradient component.

We apply our method for three different parameter combinations, obtaining in each case a polynomial expression for the potential with the same nonzero terms. The coefficients for these terms are displayed in Table I. For the nongradient cases, the decompositions are, as desired, suborthogonal.

\section{Bounds on the quasipotential}

A useful property of the (sub-)orthogonal decomposition is that it may be used to obtain predicted MAPs. As discussed in Sec. II, MAPs are those paths that minimize the action functional and provide a definition of the true quasipotential. In general these paths must be found via a costly optimization that must be performed for every point in the state space. For a system that satisfies the orthogonal decomposition, however, the MAP from a fixed point $\boldsymbol{x}_{o}$ to another point $\boldsymbol{x}_{e}$ within the basin of attraction, is one that follows the ODE,

$$
\dot{\boldsymbol{x}}=\nabla U+f_{U} \text {. }
$$

Such a path may therefore readily be obtained by simulating the time reversal of (36): starting at $\boldsymbol{x}_{e}$ and following $\dot{\boldsymbol{x}}=$ $-\nabla U-f_{U}$. Furthermore, such a path is guaranteed to exist in the orthogonal case, as discussed in Appendix C.

While we expect paths described by (36) to match exactly in the orthogonal case, for suborthogonal cases we hope that there may still be close agreement, depending on the degree of orthogonality. We therefore compare the predicted and exact paths, evaluating the true MAPs via our own implementation of the optimization approach detailed in Ref. [19]. A comparison of paths from the fixed point $\boldsymbol{x}_{o}=(-1.0,0.0)$ is given in Fig. 4 . In the gradient case, the paths match exactly, as expected. For the first nongradient cases, there is clearly some disagreement, although the sense of curvature of the paths is generally the same for the predicted and true MAPs.

An alternative prediction of the MAPs may be provided by a simple forward integration of the original ODE, since this requires no decomposition of the vector field. While these predicted paths agree fairly well in some cases, in others [notably in Fig. 4(b)] the predictions are very different.

Although the SOS-predicted and true MAPs are quantitatively different, these paths may still be used to provide an estimate of the true quasipotential. Given that the quasipotential is defined in terms of the infimum of the action over all possible paths, the action of any path that we choose can provide an upper bound to the true quasipotential. While most choices of possible path will give an action much greater than that of the MAP, the SOS predicted path may be expected to give a tighter bound, owing to the qualitative similarity with the true MAP. In addition to this upper bound on the true quasipotential, the potential $U$ itself provides a lower bound, as discussed in Sec. II.

A quantitative comparison between the true quasipotential $Q$, the potential from the decomposition $U$ and that from the SOS predicted path $S$ is given in Fig. 5. Here, $Q$ is evaluated by computing the action of the MAP from $\boldsymbol{x}_{o}=$ $(-1.0,0.0)$ to each of the interrogated points, requiring a separate optimization to be performed for each point; $U$ is obtained directly from the decomposition of the vector field; $S$ is computed by finding a predicted path following (36) and then using the geometric minimum action method [17] to compute the action of this path.

In the gradient case there is almost exact agreement between all three methods, demonstrating that the obtained decomposition provides both the true quasipotential and predicts the correct paths. In the first nongradient case, the upper bound remains remarkably tight, despite the large differences between the true and predicted paths. For the second nongradient case the bounds are even tighter, with an exact match along the $x$ axis. In all cases, each of $U$ and $S$ can be seen to indeed be lower and upper bounds respectively.
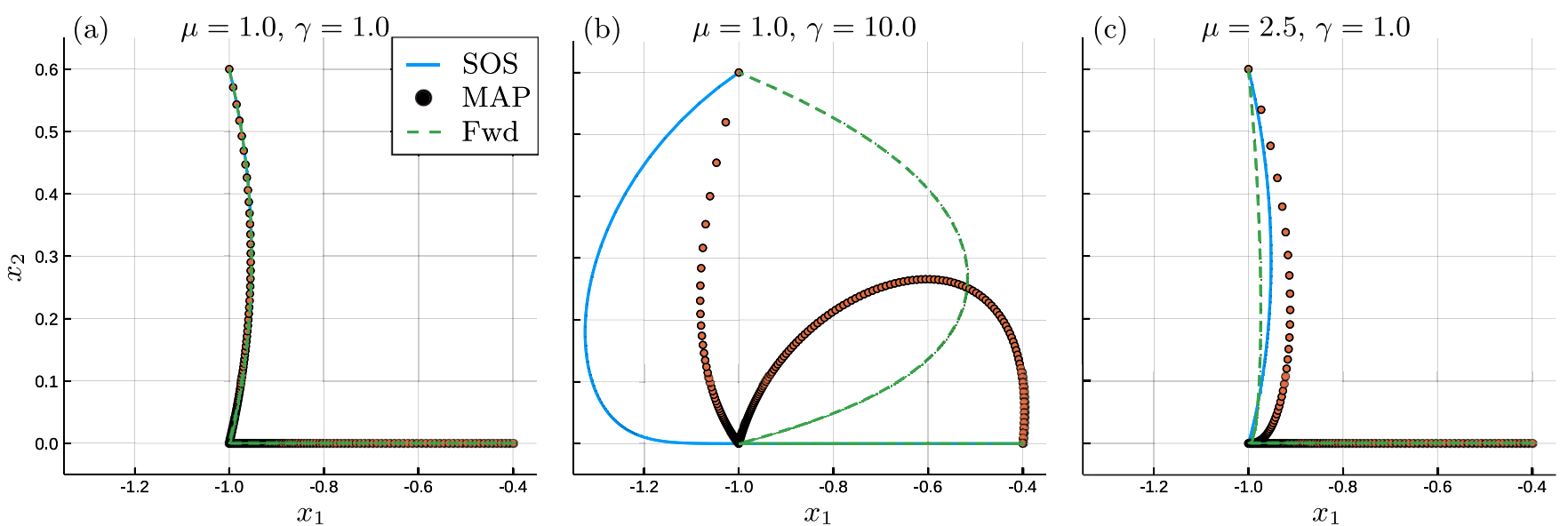

FIG. 4. A comparison of the estimated and true MAPs. Paths start at the fixed point $\boldsymbol{x}_{o}=(-1.0,0.0)$ and end at the points $\boldsymbol{x}_{e}=(-0.4,0.0)$ and $\boldsymbol{x}_{e}=(-1.0,0.6)$. Points along the minimum action paths are equally spaced in time. The SOS path is estimated by integrating $\dot{\boldsymbol{x}}=$ $-\nabla U-f_{U}$, while the "Fwd" path is obtained by integrating the original ODE $\dot{\boldsymbol{x}}=f(\boldsymbol{x})$, both starting at the end points $\boldsymbol{x}_{e}$. 

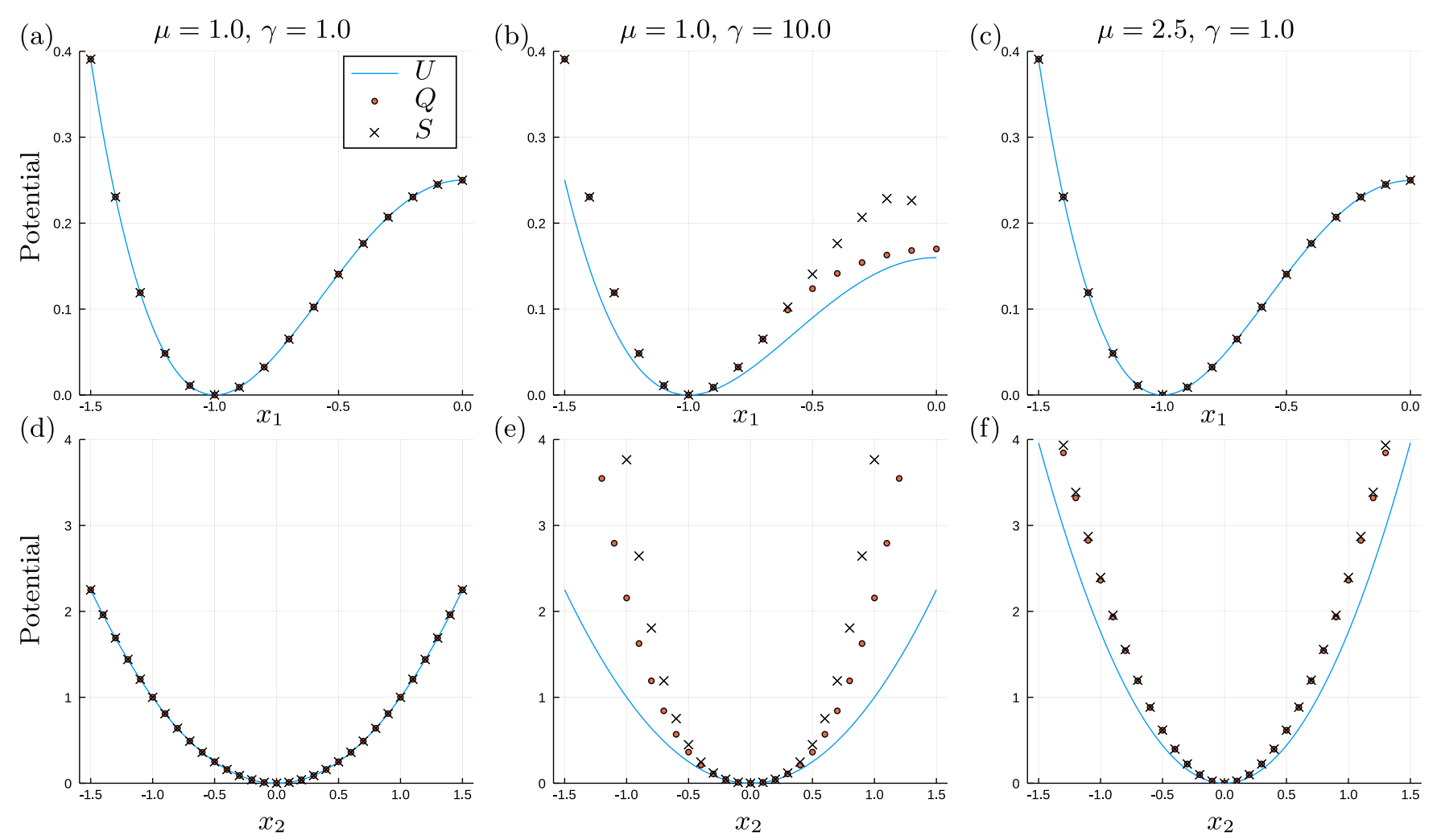

FIG. 5. A comparison of the estimates of the quasipotential. $U$ is the potential formed from the suborthogonal decomposition, $Q$ is the true quasipotential, and $S$ is the action of the SOS predicted MAP.

\section{CONCLUSIONS}

Evaluating the quasipotential for linear and nonlinear SDEs is a challenging problem, yet one with significant interest and motivation. In this work we have provided a novel method for the calculation of the quasipotential based on the sumof-squares method for constructing Lyapunov functions. Our method is applicable to systems for which the governing equations are polynomial and involves solving an optimization over the coefficients of a polynomial potential function.

The construction of an informative landscape is motivated by three key requirements. First, we would like the potential to correctly capture the basins of attraction for the deterministic system. Such a requirement is equivalent to the potential being a Lyapunov function and is therefore naturally achieved by our method. Second, it is desirable to have an estimate of the most probable transition trajectories between basins of attraction; the so-called minimum action paths. For cases permitting an orthogonal decomposition of the dynamics, the paths may readily be obtained from the two vector-field components. For cases in which the decomposition is suborthogonal, the obtained paths may provide a more-or-less accurate approximation, suitable for use as an initial guess in an optimization routine. Finally, we may wish for the potential function to be a quasipotential for the system, accurately describing the transition probabilities for situations of vanishingly small noise. Again, for systems permitting an orthogonal decomposition of the dynamics our method calculates exactly such a quasipotential, while for suborthogonal cases the potential may be used to provide both a lower and upper bound.
The first key limitation of the method is in its applicability to only polynomial systems. However, such systems are commonplace in, e.g., mass action models of chemical kinetics and linear models for dynamical systems. A closely related limitation is in the ability of the method to express the potential itself in terms of a polynomial. It is likely that in some cases, even if the governing equations are polynomial, a potential satisfying the normal decomposition must be expressed in some expanded basis beyond monomial terms. Regardless, our polynomial suborthogonal potential still provides useful bounds.

A second key limitation is that the obtained quasipotential is only valid for systems with additive noise, in which the noise tensor is equal to the identity matrix. Yet this is a common approximation, especially when the magnitude of the noise tends to zero, and furthermore can always be achieved for linear systems via a coordinate transform [51]. If multiplicative noise is present but may be expressed as a polynomial, it is possible that in some cases this could be incorporated into the algorithm; however, this is beyond the scope of this study.

While we have provided a method that generates an analytical expression for the quasipotential for a particular subset of SDE systems, it remains unclear if such a feat can be achieved in more general cases. Ultimately, we hope that the method provides another useful tool for the interpretation and analysis of stochastic dynamical systems and may pave the way for more general methods to obtain the quasipotential in a symbolic manner.

The algorithms discussed in this work have been implemented in both Julia and MATLAB, freely accessible at [56] and [57], respectively. 


\section{ACKNOWLEDGMENTS}

We are grateful to David Schnoerr for critical reading of the manuscript. R.D.B. and M.P.H.S. are funded from the BBSRC through Grant No. BB/N003608/1.

\section{APPENDIX A: OPTIMALITY IN THE LINEAR CASE}

In Sec. III B, we presented a method by which we maximize a lower bound for the quasipotential $U$, attempting to make the potential as steep as possible. Here we justify this method by proving that, in the linear case, the matrix defining a normal decomposition is also that which provides the largest potential, defined in a suitable way.

Lemma 1. Consider a full rank real matrix $A \in \mathbb{R}^{n \times n}$ whose eigenvalues have negative real part and $U(\boldsymbol{x})=\frac{1}{2} \boldsymbol{x}^{\top} P \boldsymbol{x}$ for some $P=P^{\top} \in \mathbb{R}^{n \times n}$.

Then the decomposition

$$
A \boldsymbol{x}=-\nabla U(\boldsymbol{x})+f_{U}(\boldsymbol{x}), \quad \boldsymbol{x} \in \mathbb{R}^{n},
$$

holds with $f_{U}(\boldsymbol{x})=(A+P) \boldsymbol{x}$.

Suppose further that $P$ solves the optimization problem:

$$
\begin{gathered}
\underset{P}{\operatorname{maximize}} \quad \operatorname{tr}(P) \\
\text { subject to } P=P^{\top}, \\
P(A+P) \preceq 0 .
\end{gathered}
$$

Then $P A+A^{\top} P+2 P^{2}=0$. Hence, $\nabla U \cdot f_{U}=0$.

Proof. First note that (A1) is feasible since $A$ is stable. Furthermore, for any $P$ satisfying $P(A+P) \leqslant 0$, we have

$$
C:=2 P^{2}+P A+A^{\top} P \leqslant 0 .
$$

Hence, the Ricatti equation,

$$
2 P^{2}-P(-A)-(-A)^{\top} P-C=0,
$$

has a (trivial) solution $P=P^{\top} \in \mathbb{R}^{n \times n}$. Now since $C^{\prime}=0 \geqslant$ $C$, it follows from Ref. [[58], Theorem 2.3] that the Riccati equation

$$
\begin{aligned}
& 2 X^{2}-X(-A)-(-A)^{\top} X \\
& \quad=2 X^{2}-X(-A)-(-A)^{\top} X-C^{\prime}=0
\end{aligned}
$$

has a maximal solution $X=P_{+}$, which satisfies $P_{+} \succeq P$. So, we have proved that there exists a solution $P_{+}$to

$$
2 P_{+}^{2}+P_{+} A+A^{\top} P_{+}=0
$$

which has the property that $P_{+} \succeq P$ for any feasible variable of (A1). Let $\hat{P}$ be the optimal decision variable of (A1). Then, since $P_{+}$is also feasible for (A1),

$$
\operatorname{tr}(\hat{P}) \geqslant \operatorname{tr}\left(P_{+}\right) .
$$

However,

$$
\begin{aligned}
P_{+} \succeq \hat{P} & \Rightarrow\left(P_{+}\right)_{i i} \geqslant(\hat{P})_{i i} \\
& \Rightarrow\left(P_{+}-\hat{P}\right)_{i i} \geqslant 0 .
\end{aligned}
$$

By (A2),

$$
\begin{gathered}
\operatorname{tr}\left(P_{+}-\hat{P}\right) \leqslant 0 \\
\Rightarrow \sum_{i=1}^{n} \underbrace{\left(P_{+}-\hat{P}\right)_{i i}}_{\text {all } \geqslant 0 \text { by }(\mathrm{A} 3)} \leqslant 0 \\
\Rightarrow \quad\left(P_{+}\right)_{i i}=(\hat{P})_{i i}, \quad i=1, \ldots, n .
\end{gathered}
$$

Hence, $P_{+} \succeq \hat{P}$ and both have the same diagonal entries. Therefore $P_{+}=\hat{P}$.

Consequently, the solution $\hat{P}$ to (A1) is the maximal solution to $\hat{P} A+A^{\top} \hat{P}+2 \hat{P}^{2}=0$.

Remark 1. In practice, for linear systems our full method (16) actually implements

$$
\begin{gathered}
\operatorname{maximize}_{P, c_{0},\left[\epsilon_{i}\right]} \sum_{i=1}^{n} \epsilon_{i} \\
\text { subject to } \frac{1}{2} \boldsymbol{x}^{\top} \boldsymbol{P} \boldsymbol{x}+c_{0} \geqslant \sum_{i=1}^{n} \epsilon_{i} x_{i}^{2}, \\
P(A+P) \preceq 0 .
\end{gathered}
$$

While (A4) this is not entirely equivalent to (A1), (A4b) $\Rightarrow$ $\sum_{i} \epsilon_{i} \leqslant \operatorname{tr}(P)$. The implemented method therefore provides a very close approximation that also generalizes to the nonlinear case.

\section{APPENDIX B: ITERATIVE IMPROVEMENT}

In Sec. IIID we displayed the iterative optimization approach through which an improved Lyapunov function could be obtained, given a suitable initial guess. The optimization procedure may be justified by proving the following lemma.

Lemma 2. Let $U: \mathbb{R}^{n} \rightarrow \mathbb{R}$ be fixed. Suppose that there exists $\alpha \geqslant 0$ and $V: \mathbb{R}^{n} \rightarrow \mathbb{R}$ such that

$$
\begin{aligned}
V & \geqslant 0, \\
M_{V} & \succeq 0, \\
\nabla V \cdot(f+2 \nabla U) & \geqslant \alpha(f \cdot \nabla U)+(1+\alpha)\|\nabla U\|^{2} .
\end{aligned}
$$

Then

$$
\alpha\left(\nabla U \cdot f_{U}\right)+\|\nabla V-\nabla U\|^{2} \leqslant \nabla V \cdot f_{V} \leqslant 0 .
$$

Proof. First lower bound $\nabla V \cdot f_{V}$ :

$$
\begin{aligned}
\nabla V \cdot f_{V}= & \nabla V \cdot(f+\nabla V) \\
= & \nabla V \cdot(f+2 \nabla U)+\|\nabla V\|^{2}-2 \nabla V \cdot \nabla U \\
(\text { by }(\mathrm{B} 1 \mathrm{c})) \geqslant & \alpha\left(f \cdot \nabla U+\|\nabla U\|^{2}\right)+\|\nabla U\|^{2} \\
& +\|\nabla V\|^{2}-2 \nabla V \cdot \nabla U \\
= & \alpha\left(\nabla U \cdot f_{U}\right)+\|\nabla U-\nabla V\|^{2} .
\end{aligned}
$$

That $\nabla V \cdot f_{V} \leqslant 0$ follows from (B1b).

Remark 2. (i) If $U$ satisfies the standard Lyapunov conditions $U \geqslant 0, M_{U} \succeq 0$, then the choice $(\alpha=1, V=U)$ also satisfies conditions (B1). Hence, there is always a feasible point which satisfies these inequalities. 
(ii) If $(\alpha, V)$ satisfiers the constraints with $0<\alpha<1$, then

$$
\alpha\left(\nabla U \cdot f_{U}\right)+\|\nabla V-\nabla U\|^{2} \leqslant \nabla V \cdot f_{V} \leqslant 0
$$

indicates that an improved lower bound has been found for $V \cdot f_{V}$. The improvement over $U \cdot f_{U}$ is determined by the difference $\|\nabla V-\nabla U\|^{2}$ and the scaling factor $\alpha$. reads

(iii) If $\alpha=0$ and $V$ satisfies the constraints, then (B1c)

$$
\|\nabla U-\nabla V\|^{2} \leqslant \nabla V \cdot f_{V} \leqslant 0,
$$

which forces $V=U$ and indicates that $U \cdot f_{U}=0$, i.e., the original Lyapunov function was a good choice.

\section{APPENDIX C: THE EXISTENCE OF PREDICTED MINIMUM ACTION PATHS}

In Sec. II A we claimed that in the case of the orthogonal decomposition, the minimum action paths within a basin of attraction would be solutions to the ODE $\dot{x}=\nabla U+f_{U}$. Furthermore, in Sec. VC we claimed that solutions to this ODE also provided a good prediction of the MAP in the case that the decomposition is suborthogonal. A key question therefore concerns the conditions under which such paths are guaranteed to exist. This is addressed by the following lemma.

Lemma 3. Consider a stable fixed point $\boldsymbol{a}$ of the ODE $\dot{\boldsymbol{x}}=$ $f(\boldsymbol{x})$, and a nearby point $\boldsymbol{b}$, where $f(\boldsymbol{x})=-\nabla U(\boldsymbol{x})+f_{U}(\boldsymbol{x})$. There exists a solution to the ODE,

$$
\dot{\boldsymbol{x}}=\nabla U(\boldsymbol{x})+f_{U}(\boldsymbol{x}),
$$

starting at $\boldsymbol{a}$ and ending at $\boldsymbol{b}$, provided that $\boldsymbol{b}$ lies in a region of the state space in which the following conditions hold:

$$
\begin{aligned}
\nabla U \cdot f_{U} & >-\|\nabla U\|^{2}, \\
U(\boldsymbol{a}) & =0, \\
U & >0 .
\end{aligned}
$$

Proof. Consider the ODE,

$$
\dot{\boldsymbol{x}}=-\nabla U(\boldsymbol{x})-f_{U}(\boldsymbol{x}) .
$$

We may use the candidate Lyapunov function $U$ to prove that a solution to this ODE, starting at $\boldsymbol{b}$ will converge to the fixed point $\boldsymbol{a}$. By conditions $(\mathrm{C} 2 \mathrm{~b})$ and $(\mathrm{C} 2 \mathrm{c}), U$ is a positive definite function for which $\boldsymbol{a}$ is a minima. We additionally require

$$
\begin{aligned}
\dot{U} & =\nabla U \cdot \dot{\boldsymbol{x}} \\
& =-\|\nabla U\|^{2}-\nabla U \cdot f_{U}<0,
\end{aligned}
$$

which is satisfied by (C2a). Given an initial condition $\boldsymbol{x}(0)=\boldsymbol{b}$, $\boldsymbol{x}(t)$ will therefore follow a path that converges to $\boldsymbol{a}$ as $t \rightarrow \infty$.

Now note that a path from $\boldsymbol{b}$ to $\boldsymbol{a}$ following (C3) is simply the time reversal of a path from $\boldsymbol{a}$ to $\boldsymbol{b}$ following (C1). Conditions (C2) are therefore sufficient for this path to exist.

Remark 3. Condition (C2a) implies that for the case of an orthogonal decomposition of the dynamics, a MAP following (C1) is guaranteed to exist. For the suborthogonal case, the requirement may be interpreted as meaning that the deviation from orthogonality must not be too strong.

As mentioned in Sec. V, the time-reversed equation (C3), actually provides a suitable method for calculating the predicted MAP via a straightforward simulation.
[1] L. Gammaitoni, P. Hänggi, P. Jung, and F. Marchesoni, Rev. Mod. Phys. 70, 223 (1998).

[2] C. Gardiner, Stochastic Methods: A Handbook for the Natural and Social Sciences (Springer, Berlin, 2009).

[3] A. Einstein, Ann. Phys. (Leipzig) 322, 549 (1905).

[4] T. Dauxois, F. Di Patti, D. Fanelli, and A. J. McKane, Phys. Rev. E 79, 036112 (2009).

[5] D. Schnoerr, G. Sanguinetti, and R. Grima, J. Phys. A 50, 093001 (2017).

[6] A. J. McKane and T. J. Newman, Phys. Rev. Lett. 94, 218102 (2005).

[7] P. C. Bressloff, Phys. Rev. E 82, 051903 (2010).

[8] D. Fanelli, F. Ginelli, R. Livi, N. Zagli, and C. Zankoc, Phys. Rev. E 96, 062313 (2017).

[9] S. di Santo, P. Villegas, R. Burioni, and M. A. Muñoz, J. Stat. Mech. (2018) 073402.

[10] G. Rigas, A. S. Morgans, R. D. Brackston, and J. F. Morrison, J. Fluid Mech. 778, R2 (2015).

[11] R. D. Brackston, J. M. Garcìa de la Cruz, A. Wynn, G. Rigas, and J. F. Morrison, J. Fluid Mech. 802, 726 (2016).

[12] P. G. Wolynes, J. N. Onuchic, and D. Thirumalai, Science 267, 1619 (1995).

[13] C. H. Waddington, The Strategy of the Genes: A Discussion of Some aspects of Theoretical Biology (Allen \& Unwin, London, 1957).
[14] N. Moris, C. Pina, and A. M. Arias, Nature Rev. Gen. 17, 693 (2016).

[15] M. I. Freidlin and A. D. Wentzell, Random Perturbations of Dynamical Systems, 3rd ed. (Springer, Heidelberg, 2012).

[16] J. Wang, K. Zhang, and E. Wang, J. Chem. Phys. 133, 125103 (2010).

[17] M. Heymann and E. Vanden-Eijnden, Commun. Pure Appl. Math. 61, 1052 (2008).

[18] D. Liu, J. Comput. Phys. 227, 8672 (2008).

[19] R. de la Cruz, R. Perez-Carrasco, P. Guerrero, T. Alarcon, and K. M. Page, Phys. Rev. Lett. 120, 128102 (2018).

[20] A. Souza and M. Tao, arXiv:1801.05776.

[21] Z. Chen and M. Freidlin, Stoch. Dyn. 05, 569 (2005).

[22] M. K. Cameron, Phys. D: Nonlin. Phenom. 241, 1532 (2012).

[23] D. Dahiya and M. K. Cameron, J. Sci. Comput. 75, 1351 (2017).

[24] M. K. Cameron, arXiv:1801.00327.

[25] J. Wang, Adv. Phys. 64, 1 (2015).

[26] P. Zhou and T. Li, J. Chem. Phys. 144, 094109 (2016).

[27] J. Wang, L. Xu, and E. Wang, Proc. Natl. Acad. Sci. USA 105, 12271 (2008).

[28] C. Lv, X. Li, F. Li, and T. Li, PLoS ONE 9, e88167 (2014).

[29] H. Ge, H. Qian, and X. S. Xie, Phys. Rev. Lett. 114, 078101 (2015).

[30] C. Li, T. Hong, and Q. Nie, Phys. Chem. Chem. Phys. 18, 17949 (2016). 
[31] J. Wang, L. Xu, E. Wang, and S. Huang, Biophys. J. 99, 29 (2010).

[32] C. Li, E. Wang, and J. Wang, PLoS ONE 6, e17888 (2011).

[33] J. Guo, F. Lin, X. Zhang, V. Tanavde, and J. Zheng, Bioinf. 33, 1583 (2017).

[34] R. Yuan, Y. Tang, and P. Ao, Front. Phys. 12, 120201 (2017).

[35] P. Ao, J. Phys. A 37, L25 (2004).

[36] L. Yin and P. Ao, J. Phys. A 39, 8593 (2006).

[37] J. X. Zhou, M. D. S. Aliyu, E. Aurell, and S. Huang, J. R. Soc. Interface 9, 3539 (2012).

[38] F. Bouchet and J. Reygner, Ann. Henri Poincaré 17, 3499 (2016).

[39] M. Tao, Phys. D: Nonlin. Phenom. 363, 1 (2018).

[40] J. Jost, Dynamical Systems (Springer, Berin, 2005).

[41] R.-S. Yuan, Y.-A. Ma, B. Yuan, and P. Ao, Chin. Phys. B 23, 010505 (2014).

[42] P. A. Parrilo, Structured Semidefinite Programs and Semialgebraic Geometry Methods in Robustness and Optimization, Ph.D. thesis, Caltech 2000.

[43] I. Dunning, J. Huchette, and M. Lubin, SIAM Rev. 59, 295 (2017).

[44] B. Legat, J. Huchette, T. Kelman, and E. Saba, JuliaOpt/SumOfSquares.jl v0.2.0, http://doi.org/10.5281/zenodo. 1208672.

[45] A. Papachristodoulou, J. Anderson, G. Valmorbida, S. Prajna, P. Seiler, and P. A. Parrilo, arXiv:1310.4716.
[46] D. Silk, P. D. W. Kirk, C. P. Barnes, T. Toni, A. Rose, S. Moon, M. J. Dallman, and M. P. H. Stumpf, Nature Commun. 2, 489 (2011).

[47] K. G. Murty and S. N. Kabadi, Math. Programm. 39, 117 (1987).

[48] F. Zhang, The Schur Complement and Its Applications, Numerical Methods and Algorithms (Springer, Boston, MA, 2005).

[49] A. Papachristodoulou and S. Prajna, in Proceedings of the 41st IEEE Conference on Decision and Control, Vol. 3 (IEEE, Las Vegas, USA, 2002), pp. 3482-3487.

[50] A. Majumdar, A. A. Ahmadi, and R. Tedrake, in Proceedings of the 53rd IEEE Conference on Decision and Control (IEEE, 2014), pp. 394-401.

[51] C. Kwon, P. Ao, and D. J. Thouless, Proc. Natl. Acad. Sci. USA 102, 13029 (2005).

[52] M. G. Neubert and H. Caswell, Ecology 78, 653 (1997).

[53] M. G. Neubert, H. Caswell, and J. D. Murray, Math. Biosci. 175, 1 (2002).

[54] M. Asllani and T. Carletti, Phys. Rev. E 97, 042302 (2018).

[55] R. S. Maier and D. L. Stein, J. Stat. Phys. 83, 291 (1996).

[56] https://github.com/rdbrackston/SDEtools

[57] https://github.com/rdbrackston/normalSoS

[58] I. Gohberg, P. Lancaster, and L. Rodman, SIAM J. Control Optim. 24, 1323 (1986). 